\title{
HIGH-FREQUENCY INSTABILITIES IN ROCKET THRUST CHAMBERS: AN ENGINEERING APPROACH
}

\author{
F. A. Fassl \\ Astrium GmbH, Business Division Launcher Propulsion \\ München 81663, Germany
}

The prediction and prevention of high-frequency (HF) instabilities in rocket engines remain challenging tasks not only for science to understand the ruling processes and to measure, simulate, and model them, but also for industry to find pragmatic ways of combining existing knowledge and experimental experience from laboratory or industrial full-scale tests and applying them systematically already at the early stages of rocket engine development programs. The French-German REST program constitutes a scientific platform aiming at combining both scientific investigation and industrial viewpoint. The concept of Astrium's stability analysis tool STABAN was elaborated in REST and systematically developed and adjusted. It constitutes an engineering approach in the frequency domain enabling quick applicability to any engine hardware. At future REST phases, the main focus will be to validate STABAN against scientific and industrial test data and to classify thrust chambers into instability risk families.

\section{NOMENCLATURE}

$\begin{array}{ll}a & \text { sound velocity, } \mathrm{m} / \mathrm{s} \\ A & \text { sectional area, } \mathrm{m}^{2} \\ A_{\mathrm{nm}} & \text { factor for additional driving terms } \\ c^{*} & \text { characteristic velocity, } \mathrm{m} / \mathrm{s} \\ C & \text { capacitance, } \mathrm{m} \cdot \mathrm{s}^{2} \\ d & \text { droplet diameter, } \mathrm{m} \\ D & \text { additional driving term } \\ G & \text { combustion gain } \\ l^{*} & \text { characteristic length, } \mathrm{m} \\ m & \text { mass, } \mathrm{kg} \\ \mathrm{Ma} & \text { Mach number }\end{array}$

This is an Open Access article distributed under the terms of the Creative Commons Attribution-Noncommercial License 3.0, which permits unrestricted use, distribution, and reproduction in any noncommercial medium, provided the original work is properly cited. 


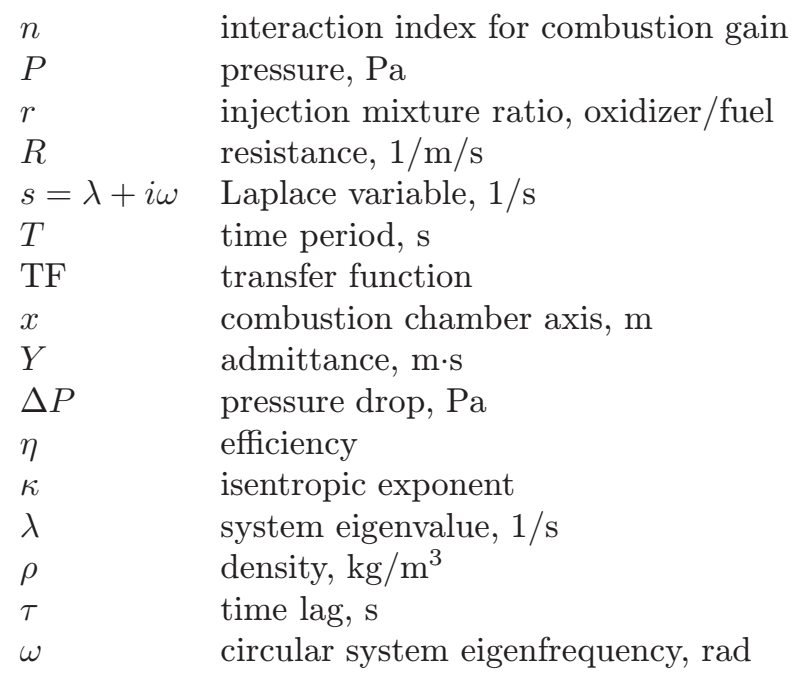

\section{Subscripts}

$\begin{array}{ll}\text { abs } & \text { absorber } \\ c & \text { combustion chamber or hot gas } \\ \text { DD } & \text { droplet drag } \\ \text { drop } & \text { propellant droplet } \\ \text { dyn } & \text { dynamic portion } \\ \text { fu } & \text { fuel } \\ \text { inj } & \text { at injection } \\ \text { ms } & \text { mass storage } \\ \text { ni } & \text { nozzle inlet } \\ \text { ox } & \text { oxidizer } \\ \text { Pc } & \text { chamber-pressure related } \\ \text { r } & \text { real part } \\ \text { rel } & \text { normalized } \\ \text { s } & \text { sensitive } \\ \text { t } & \text { total } \\ \text { vc } & \text { gas-velocity related }\end{array}$

\section{INTRODUCTION}

An extensive experimental data base exists at Astrium from the past decades of thrust chamber testing for ARIANE 5. This data base encompasses a large variety of thrust chambers extending from subscale testing on quasi-scientific 
level to full-scale development, acceptance, and qualification tests, and that for hypergolic, green, and cryogenic propellants with different injection system designs and operation conditions. This enables a systematic exploitation for trend analyses and for extracting a family behavior for an early definition of the development direction for future thrust chamber designs.

The French-German REST group, dedicated to an improved understanding of processes leading to HF instability in rocket engines, follows the derivation of physical correlations for significant stability parameters and processes and supports the development, testing and validation of predictive tools for industrial use. Experimental data are created in laboratory scale completing the industrial test data bases and the engineering knowledge.

In the scope of REST, the STABAN code is developed at Astrium for systematic analyses of experimental data for a stability-related data base as well as for predictions of future engine concepts or for extrapolations from subscale to full-scale hardware. The modeling concept and some practical examples of application are presented.

\section{MODELING APPROACH}

Figure 1 depicts the main players in stability physics which are considered in STABAN. Shown in Fig. $1 a$ is the hardware of one of Astrium's thrust chambers. All system parts connected to the combustion chamber are represented by their admittances $Y$ (Fig. 1b). The dynamic impact of the propellants at

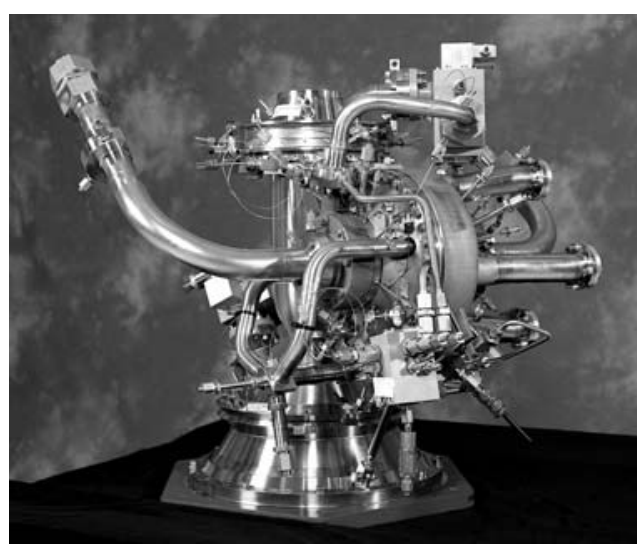

(a)

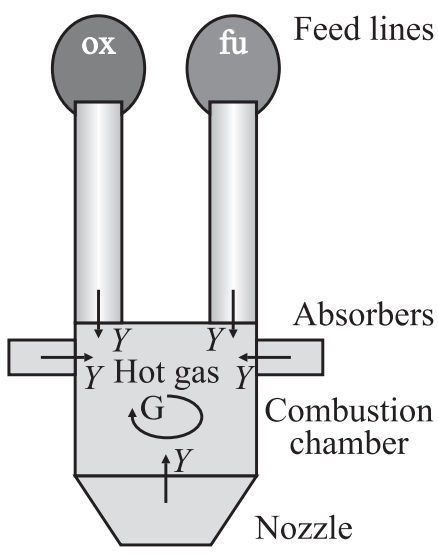

(b)

Figure 1 Coupled system stability model 
the time of injection is considered as well as the one of the convergent part of the exhaust nozzle. Admittance is also provided by intentionally incorporated damping devices. An intrinsic admittance is due to the droplet drag from the evaporating propellants. The combustion gain $G$ acts against these admittances as the main driving process for instability initiation. It describes the dynamic coupling of the combustion chamber pressure with the provision of ignitable propellant gas. All other intrinsic physical phenomena in the combustion chamber are combined in the additional driving terms $D$ for oxidizer and fuel (see Eq. (2) below).

\subsection{System Model}

A general propulsion system which can be tailored to the actual conditions and reduced to the effective system parts by appropriately modifying the submodels and the model parameters is described by:

$$
G_{\text {rel }}+D_{\text {ox.rel }}+D_{\text {fu.rel }}=Y_{\text {rel }}
$$

where

$$
\begin{aligned}
D_{\text {ox.rel }} & =A_{n m} \frac{\bar{r}}{\bar{r}+1} \frac{\bar{P}_{c}}{2 \Delta \bar{P}_{\text {inj.ox }}}\left(1+k_{\mathrm{ox}}\right)\left(1+s \Theta_{\mathrm{ox}}\right) e^{-\bar{\tau}_{\mathrm{t} . \mathrm{ox}} s} Y_{\text {inj.ox.rel }} \\
D_{\text {fu.rel }} & =A_{n m} \frac{1}{\bar{r}+1} \frac{\bar{P}_{c}}{2 \Delta \bar{P}_{\text {inj.fu }}}\left(1+k_{\mathrm{fu}}\right)\left(1+s \Theta_{\mathrm{fu}}\right) e^{-\bar{\tau}_{\mathrm{i} . \mathrm{fu}} s} Y_{\text {inj.fu.rel }} \\
Y_{\text {rel }} & =Y_{\text {ms.rel }}+Y_{\text {Pc.rel }}+Y_{\text {vc.rel }}+Y_{\mathrm{DD} . \text { rel }}+Y_{\text {abs.rel }} .
\end{aligned}
$$

The intrinsic physics of the combustion chamber and the effects from its interfaces with the surrounding system are arranged such that all processes having the potential to drive instability are combined on the left side and all processes supposed to damp are on the right side of Eq. (1). They are the combustion gain $G_{\text {rel }}$, the additional driving terms $D_{\text {rel }}$ for oxidizer and fuel, respectively, and the damping admittances $Y_{\text {rel }}$. Table 1 summarizes all terms in Eqs. (1)-(3) which are based on fundamental considerations [1].

Equation (1) is generally valid, still, low-frequency (LF) and HF oscillations must be differently treated to account for the different physical impacts of the processes. Table 1 distinguishes between the linear and the nonlinear oscillation cases, the LF and the HF ranges, and the oscillation modes. Thereby, TxRx means pure transversal modes (tangential and/or radial) and TxRxLx stands for longitudinal or combined modes (longitudinal mode with/without transversal modes). The terms in Table 1 contain normalized quantities which are indicated by a roof symbol. The normalization rules are basically adopted from [1].

In line 1.1 of Table 1, the pressure-dependent (linear) combustion gain is given which is only needed for HF investigations. This derives from the fact 


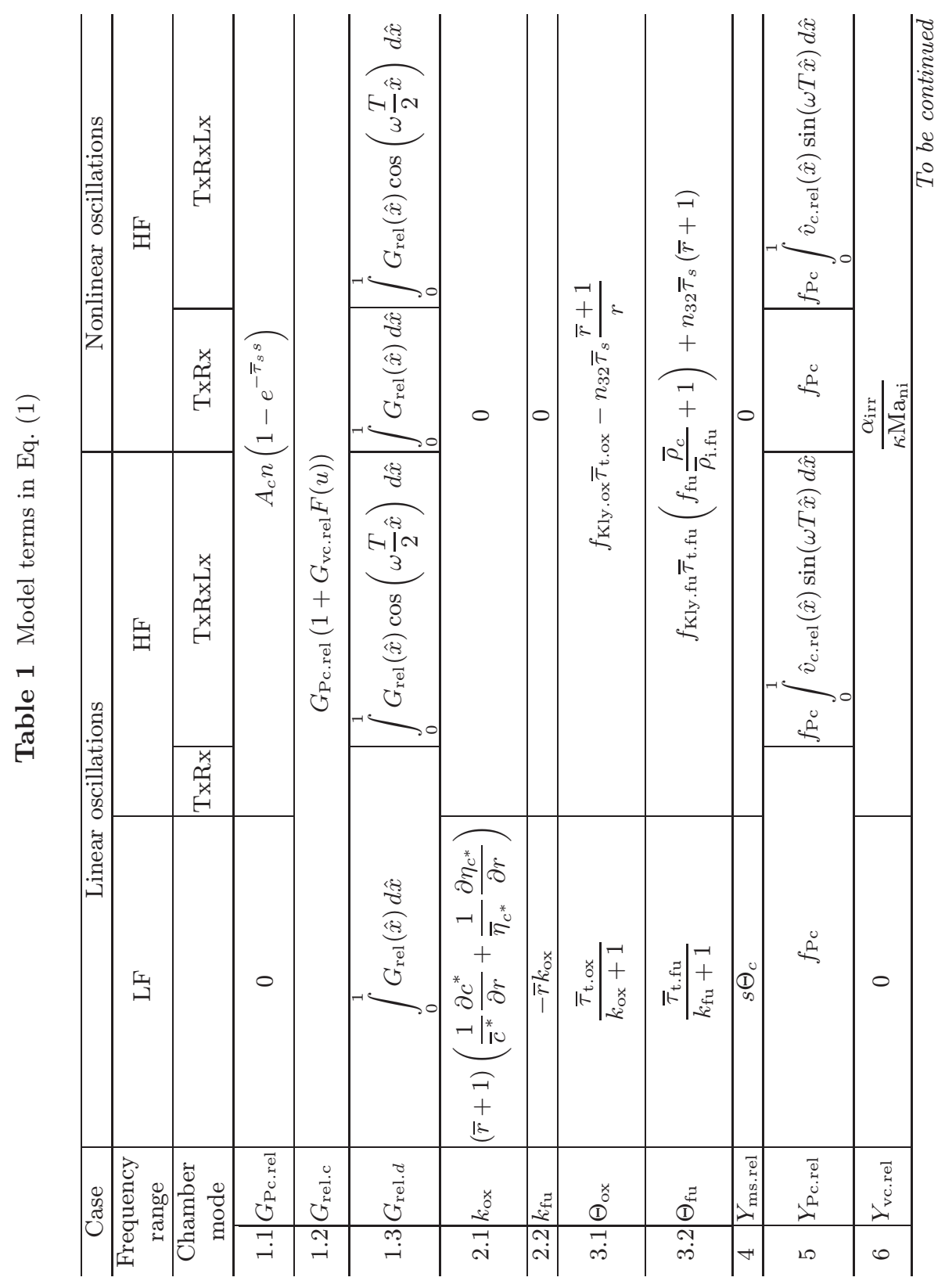




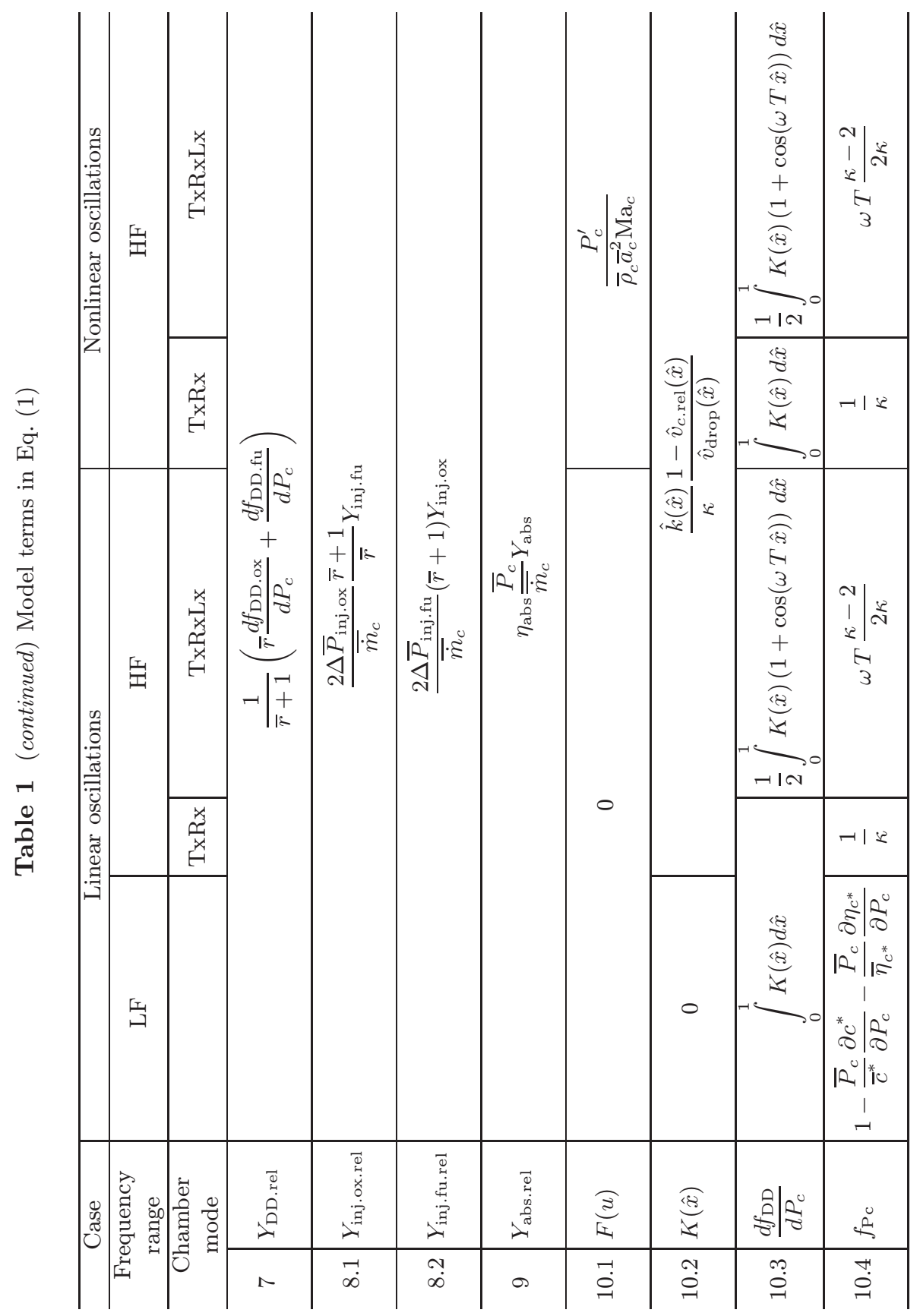


that the combustion chamber is treated as a black box for LF analyses without the need for resolving intrinsic physics. The linear combustion gain is extended by a nonlinear term to consider the effect of flow velocity variation. Since flow velocity is a three-dimensional (3D) quantity and STABAN is not capable of spatially resolving the combustion chamber, the parameter $G_{\mathrm{vc} . \mathrm{rel}}$ must be empirically determined from test data, typically from time intervals with limit-cycle oscillations of the com-

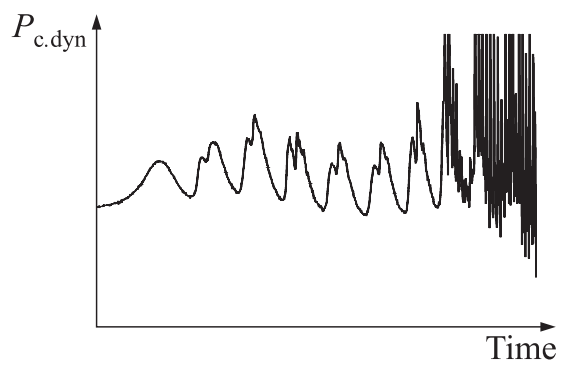

Figure 2 High-frequency instability initiation by LF oscillation bustion chamber pressure. The ampli-

fication factor $F(u)$ in line 10.1 is only calculated when a critical value of the gas velocity amplitude is surpassed; otherwise, it is zero. In Fig. 2, a similar case is presented for a critical pressure amplitude level only beyond which nonlinear instability is initiated. The nonlinear HF instability is characterized by an asymmetric oscillation around the LF variation.

To practically decide (in terms of the system transfer function) whether $F(u)$ is to be considered or not, the linear balance $B_{\text {rel.r }}$ between the real parts of the left- and right-hand sides of Eq. (1) is calculated at each time of system operation. According to Table 1, Eq. (1) can be rearranged as follows:

$$
0=\frac{B_{\text {rel.r }}-G_{\text {Pc.rel.r }} G_{\mathrm{vc.rel}} F(u)}{1+D_{\text {vc.rel }} F(u)}
$$

with

$$
B_{\text {rel.r }}=Y_{\text {rel.r }}-D_{\text {ox.rel.r }}-D_{\text {fu.rel.r }}-G_{\text {Pc.rel.r }} .
$$

In case $B_{\text {rel.r }}$ is zero, $F(u)$ must be also zero, i. e., nonlinear contribution is impossible. This also holds for $B_{\text {rel.r }}$ greater than zero, since then the damping is higher than the driving terms and no instability is present at all. Only at negative $B_{\text {rel.r }}$, additional nonlinear effects are likely to occur. As stated further up, the model parameter $G_{\mathrm{vc} . r e l}$ is determined by model adaptations to limitcycle oscillations (Table 2) in order to consider the right order of magnitude of the nonlinear contribution in case $F(u)$ is not zero.

Lines 1.1 and 1.2 (see Table 1 ) only hold, if a condensed $\left(G_{\text {rel.c }}\right)$ combustion is presumed in a flame front below the faceplate. If combustion is distributed along the combustion chamber axis $\left(G_{\text {rel.d }}\right)$, line 1.3 becomes relevant to get the integral value with the longitudinal chamber-mode shape of a closed-closed oscillator. In STABAN, the distributed combustion is not considered yet. This is just an option for a future model extension, since the condensed combustion 
Table 2 Essential model parameters in Eq. (1)

\begin{tabular}{l|c|c|c|c}
\hline \multicolumn{2}{c|}{ Oscillation case } & \multicolumn{2}{|c|}{ Linear oscillations } & Nonlinear oscillations \\
\hline \multicolumn{2}{|c|}{ Frequency range } & LF & HF & HF \\
\hline 1.1 & $\tau_{\text {t.ox }}$ & output & \multicolumn{2}{c}{ input } \\
\hline 1.2 & $\tau_{\text {t.fu }}$ & output & & input \\
\hline 2.1 & $A_{c}$ & 0 & output & input \\
\hline 2.2 & $n$ & 0 & & output \\
\hline 2.3 & $\tau_{\mathrm{s}}$ & 0 & & input \\
\hline 2.4 & $G_{\text {vc.rel }}$ & \multicolumn{2}{|c}{ input } \\
\hline 3.1 & $f_{\text {Kly.ox }}$ & 1 & output & input \\
\hline 3.2 & $f_{\text {Kly.ox }}$ & 1 & output & input \\
\hline 3.3 & $f_{\text {fu }}$ & 0 & \multicolumn{3}{|c}{ input } \\
\hline 3.4 & $n_{32}$ & \multicolumn{3}{|c}{} \\
\hline 4 & $d_{\text {drop }}$ & \multicolumn{3}{|c}{} \\
\hline 5 & $\eta_{\text {abs }}$ & 0 & \multicolumn{3}{|c}{} \\
\hline
\end{tabular}

constitutes the worst case for instability initiation [2] and, thus, for stability margin calculations as well. At distributed combustion, the different contributions of the forward and backward pressure waves in case of longitudinal oscillation modes lead to additional damping. This also holds for transversal modes (but much less) due to the superposed mean flow.

In Eq. (2), the parameters $k_{\mathrm{ox}}$ and $k_{\mathrm{fu}}$ appear. They correspond to the steadystate gas mass flow (see Eq. (6) below) and are, therefore, only applicable to the LF oscillation case for which the combustion chamber is considered as a black box (see Table 1, lines 2.1 and 2.2). For the LF case, Eq. (6) also delivers $Y_{\text {Pc.rel }}$ (line 5 in Table 1 ) by differentiation. $Y_{\text {vc.rel }}$ (line 6 in Table 1 ) is zero, since intrinsic combustion chamber effects are not relevant to the LF case.

Whereas the factors $\left(1+k_{\mathrm{ox}}\right)$ and $\left(1+k_{\mathrm{fu}}\right)$ as well as the admittances $Y_{\mathrm{Pc} . \mathrm{rel}}$ and $Y_{\text {vc.rel }}$ consider the impact of the hot gas flow in the combustion chamber and the convergent nozzle part, the factors $\left(1+s \Theta_{\mathrm{ox}}\right)$ and $\left(1+s \Theta_{\mathrm{fu}}\right)$ in Eq. (2) symbolize the impact of pulsing mass accumulation in the combustion chamber originating from the following physical processes:

- varying total combustion time lags making quicker droplets surpass slower ones (Klystron effect);

- varying sensitive time lag leading to a pulsing combustion; and

- pulsing coolant film consumption (only in case the combustion chamber wall is film cooled).

In lines 3.1 and 3.2 (see Table 1), the terms $\Theta_{\text {ox }}$ and $\Theta_{\text {fu }}$ in Eq. (2) are given for the oxidizer and fuel contribution. The differences for the LF and HF oscillation cases result from two reasons: 
(1) the intrinsic effects, pulsing combustion, and coolant film consumption are neglected (but Klystron effect is fully considered; see Table 2, lines 3.1 and 3.2) also for the LF case; and

(2) in the steady-state nozzle, Eq. (6) cannot be applied for the HF case, since this equation constitutes a bulk approach purely related to pressure without the velocity impact expressed by the complex exhaust nozzle admittance [3]. This can only be considered in Eq. (7) below.

The admittances $Y_{\text {inj.ox.rel }}$ and $Y_{\text {inj.fu.rel }}$ in Eq. (2) are determined in lines 8.1 and 8.2 (see Table 1). They contain the propellant injection admittances related to the outlet areas of the injection elements. The propellant feed lines are described by series connections of elbows, bellows, pumps, turbines, throttles, and straight tubes. The series connections are mathematically realized by a multiplication of the individual transfer matrices opposite to the flow direction. The injection elements are treated as the last tubes of the propellant feed lines:

$$
\left(\begin{array}{c}
\dot{m}^{\prime} \\
P^{\prime}
\end{array}\right)_{\mathrm{inj}}=\prod_{i=1}^{n} A_{n+1-i}\left(\begin{array}{c}
\dot{m}^{\prime} \\
P^{\prime}
\end{array}\right)_{t a n k}=B\left(\begin{array}{c}
\dot{m}^{\prime} \\
P^{\prime}
\end{array}\right)_{\mathrm{tank}}
$$

When applying the tank boundary condition, the injection admittance results in

$$
Y_{\mathrm{inj}}=-\frac{b_{12}}{b_{11}} .
$$

In case of complex feed lines, particularly, for LF analyses, the matrix coefficients can also become quite complex. For HF cases, the limitation to the injection elements is often sufficient to simplify the matrix coefficients to the physics of one straight tube. This determination procedure is identically applied for the oxidizer and fuel lines.

The admittance $Y_{\text {ms.rel }}$ in line 4 (see Table 1) from Eq. (3) describes the capability of the combustion chamber to dynamically store mass. The technical measure of storage capability is the residence time $\Theta_{c}$ of the gas in the combustion chamber:

$$
\Theta_{c}=\frac{m_{c}}{\dot{m}_{c}}=\frac{l^{*} c^{*} \eta_{c^{*}}}{a^{2}} .
$$

Similar to the terms $s \Theta_{\text {ox }}$ and $s \Theta_{\text {fu }}$ in Eq. (2), $Y_{\text {ms.rel }}$ also constitutes an LF effect. Therefore, it is set to zero for HF oscillations or, as an option, it is to be multiplied by an empirical correction factor similar to $f_{\text {Kly.ox }}$ and $f_{\text {Kly.fu }}$ for the Klystron effect (see Tables 1 and 2, lines 3.1 and 3.2).

This admittance is directly proportional to the frequency, i. e., the higher the frequency, the higher the damping capability of the combustion chamber volume. This is the reason why combustion chambers with big volumes or small propellant 
mass flow rates are unlikely to become unstable, provided the disturbances from the feed lines are not too severe (admittances $Y_{\text {inj.ox.rel }}$ and $Y_{\text {inj.fu.rel }}$ ).

The next two admittances $Y_{\text {Pc.rel }}$ and $Y_{\text {vc.rel }}$ in lines 5 and 6 (see Table 1 ) derive from the chamber flow equation. The following equations apply (Eq. (6) for the LF case and Eq. (7) for the HF case):

$$
\begin{aligned}
& \dot{m}_{c}=\frac{A_{\mathrm{th}} P_{c}}{c^{*} \eta_{c^{*}}} ; \\
& \dot{m}_{c}=A \rho_{c} v_{c} .
\end{aligned}
$$

Both equations are linearized and Laplace transformed. For Eq. (7), the isentropic condition is additionally supposed as well as the nozzle admittance $\alpha_{\text {irr }}$ definition [3] is applied. The distributed gas velocity in the combustion chamber must be considered for $Y_{\text {Pc.rel }}$ in case of longitudinal modes that then provides an additional damping. It is noted that the pressure-related admittance $Y_{\text {Pc.rel }}$ is positive and, therefore, always stabilizing, whereas the velocity-related admittance $Y_{\text {vc.rel }}$ may also be negative depending on the value of $\alpha_{\text {irr }}$ as a function of nozzle geometry and mode shape.

The droplet drag admittance $Y_{\text {DD.rel }}$ in Eq. (3) is defined in line 7 (see Table 1) and considers the energy loss from the interaction between the propellant droplets and the combustion gas. The corresponding terms are presented in lines 10.2 and 10.3. The assumption behind is that, at each axial position in the combustion chamber, a certain amount of liquid propellant mass is present. The injected-propellant droplet sizes are determined from given experimental correlations and their consumption is determined from the relative velocity between combustion gas and droplets. To get the total droplet drag, the term given in line 10.2 is integrated along the combustion chamber axis. Since distributed velocities are considered and droplet drag is assumed to solely depend on pressure, longitudinal modes require the consideration of the mode shape leading to a reduction of damping. This is in contrast to line 1.3 where combustion gain reduction widens stability margin.

Finally, the admittance of acoustic absorbers $Y_{\text {abs.rel }}$ is defined in line 9 (see Table 1). The absorbers are treated like the feed lines for $Y_{\text {inj.rel }}$ in lines 8.1 and 8.2 (see Eq. (5)) but with the closed-end condition instead of the tank condition. The total admittance is the single absorber admittance times the number of identical absorbers. This is repeated for each absorber class and, finally, delivers $Y_{\text {abs }}$ of the total absorber ring.

It is noted that the model does not consider any spatial distribution of absorbers. This means that, particularly for tangential modes, the damping effect of the absorber ring is overestimated. This requires a correction by parameter $\eta_{\text {abs }}$. For radial and longitudinal modes, this correction is not needed, because then the absorber ring operates uniformly at the right place near the closed end of the combustion chamber. 


\subsection{Parameter Settings}

In the equations and model terms, several parameters occur which must be known from experience, model adaptation to test data, or literature. Otherwise, assumptions must be undertaken. In Table 2, the essential model parameters are introduced.

Equation (1) constitutes the balance condition between instability drivers and instability dampers. This condition can be utilized to iteratively solve its real and imaginary parts for two unknown parameters at times when measurement data also indicate such a balance. For the future, it is foreseen to implement STABAN into an optimization algorithm to simultaneously fit all parameters to a sufficient number of tests.

In lines 1.1 and 1.2 of Table 2, the total combustion time lags of oxidizer $\tau_{\text {t.ox }}$ and fuel $\tau_{\text {t.fu }}$ are depicted (see Eq. (2)). They are determined by iterating Eq. (1) for the LF case. The other model parameters take on the values as given in Table 2. These values are derived from the assumption that intrinsic effects inside the combustion chamber play no role for the black box approach, except for the Klystron effect known to be LF-related (see also Table 1, lines 3.1 and 3.2). The total combustion time lags are then available for calculations in the HF range.

In the next step, the model parameters $A_{c}$ (see Table 1, line 1.1) and $f_{\text {Kly.ox }} / f_{\text {Kly.fu }}$ (lines 3.1 and 3.2 ) are determined. The parameters $f_{\text {Kly.ox }}$ and $f_{\text {Kly.fu }}$ consider the fact that the LF-related Klystron effect would be overestimated in the HF range without correction. Typically, no difference is made for both propellants. To find reasonable estimations for the absorber ring efficiency $\eta_{\text {abs }}$ (line 9), it is recommended first to take tests without absorbers $\left(\eta_{\text {abs }}=0\right)$ or restrict consideration to radial or longitudinal modes for which absorber ring efficiency is maximum $\left(\eta_{\mathrm{abs}}=1\right)$.

The parameter $n_{32}$ (see Table 1, lines 3.1 and 3.2) considers the impact of the velocity variation on the sensitive time lag $\tau_{s}$ (line 1.1). This effect can normally be neglected compared to pressure impact, i. e., it constitutes a small value or can be even set to zero. In case when sufficient thrust-chamber test data are available with sufficient quality, statistical optimization calculations could be performed to elaborate the trends and dispersions of such intrinsic and hardly calculated model parameters. A comparable case is the combustion time delay, the validation of which is described in section 3 .

It is noted that this adaptation approach follows from practical experience. Depending on the specific case considered, any other combination of two parameters can be taken for iteration in the HF range. As an option, further adaptation calculations can follow for tangential modes to determine parameter $\eta_{\text {abs }}$ for this mode shape. The already known values of $\tau_{\mathrm{t} . \mathrm{ox}}, \tau_{\mathrm{t} \text {.fu }}$ and $f_{\mathrm{Kly} . \mathrm{ox}}=f_{\mathrm{Kly} \text {.fu }}$ are then utilized for determining the new $A_{c}$ and $\eta_{\text {abs }}$ for the tangential mode. 
Finally, the last unknown parameter $G_{\mathrm{vc} . \mathrm{rel}}$ (see Table 1, line 1.2) is determined in the $\mathrm{HF}$ range, if required and relevant for the thrust chamber operation. Equation (1) is again iterated at times when experimental data show a nonlinear balance. In this case, $A_{c}$ is also determined assuming a modified combustion gain.

The sensitive time delay $\tau_{s}$ in line 2.3 (see Table 2) is typically determined from the chamber resonance condition. The droplet sizes at injection $\left(d_{\text {drop }}\right.$ in line 4) are given by experimental correlations from the literature or laboratory tests. The parameter $f_{\text {fu }}$ in line 3.3 (see Table 1, line 3.2) gives a constant ratio of coolant flow rate to total chamber flow rate in case of film cooled combustion chambers; otherwise, it is zero. The interaction index $n$ is 0.5 for coaxial injection elements [1]. In case all other parameters in Table 2 are sufficiently known from previous adaptations for the same thrust chamber family, the effective injection droplet size $d_{\text {drop }}$ can also be determined by Eq. (1) for the HF case.

\section{MODEL VALIDATION APPROACH}

The basic approach of model parameter determination is already described in section 2. In the following, two particularly interesting aspects are highlighted in more detail.

\subsection{Total Combustion Time Lags}

In addition to the iterative approach described in section 2, another method exists for determining the combustion time lags from the experimental pressure loss oscillations across the injection elements.

Shown in the left part of Fig. $3 a$ is the time interval in which an oscillation of the relative injection pressure loss on the oxidizer side exists with constant amplitude. Consequently, resonance is given between oxidizer dome and combustion chamber. On fuel side, the oscillation amplitudes of the relative injection pressure drop are much smaller and not so clearly organized, because fuel is gaseous here.

The results of calculations for the oxidizer are presented on the right side of Fig. $3 a$. Here, the interval, shown on the left side, constitutes one single point. A noticeable dispersion around $\tau_{\mathrm{t}}=0.9 \mathrm{~ms}$ indicates that resonance frequencies correspondingly change from interval to interval. It is noted that such resonance oscillations are typically taken from combustion noise, since specific test goals for subsequent systematic and well-defined stability analyses are rarely considered and stability aspects are mostly treated only as passenger test objectives.

Figure $3 b$ introduces the way described in section 2. In this case, Eq. (1) is iterated for the LF case (see Table 2, lines 1.1 and 1.2). The mean operation 

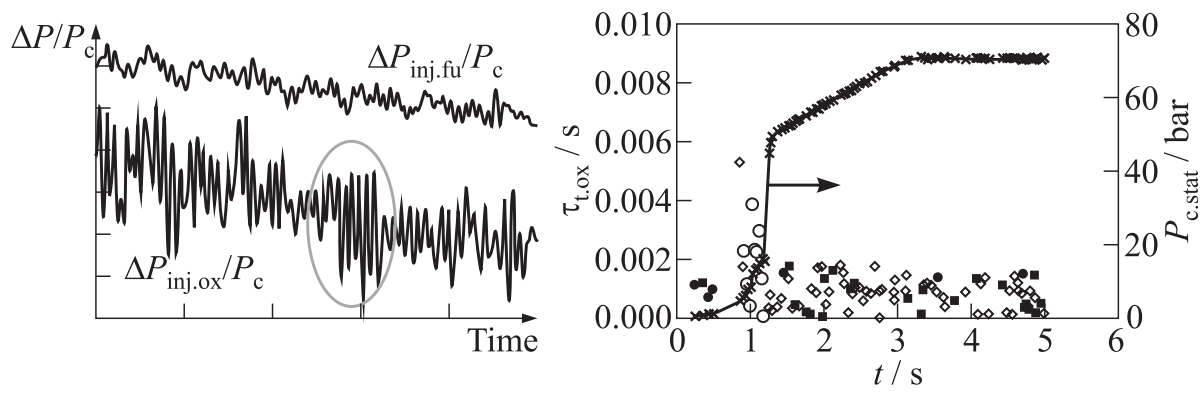

(a)
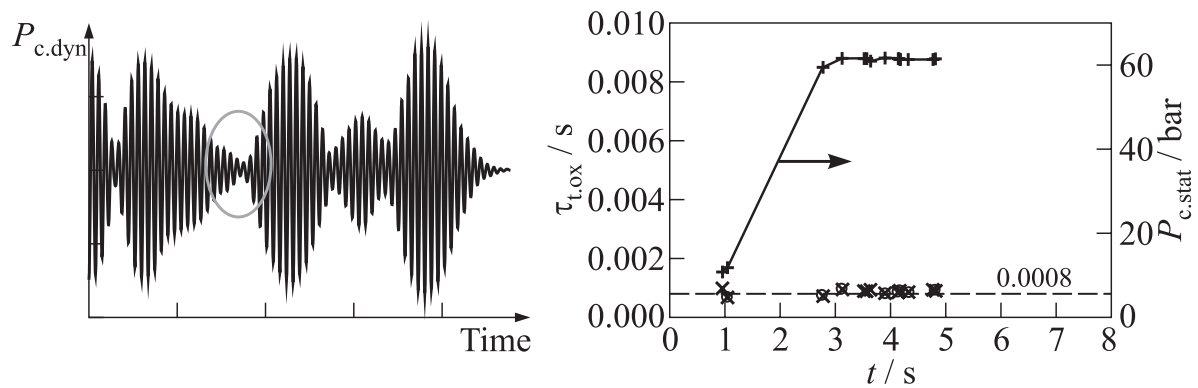

(b)

Figure 3 Determination of the total combustion time lags from pressure phase shifts $(a)$ and forced condition iteration $(b)$

conditions are calculated for each interval where the combustion-chamber pressure oscillation is zero. The results of calculations are shown on the right side of Fig. $3 b$.

The existence of two parallel calculation approaches facilitates the determination of the most probable values of these essential but hidden quantities. Here, the iteration approach confirms the mean value in Fig. $3 a$. As a further aspect, the mathematical parameter can be proven to be consistent with the physical quantity.

\subsection{Absorber Parameters}

Three parameters are to be considered for absorber model validation. They are the resistance factor $\Gamma_{\text {abs }}$, the effective fluid temperature $T_{\text {abs }}$ inside the absorber, and the absorber length correction $\Delta l_{\text {abs }}$ for a theoretical absorber extension into the combustion chamber to account for flow coupling effects (Fig. 4). 

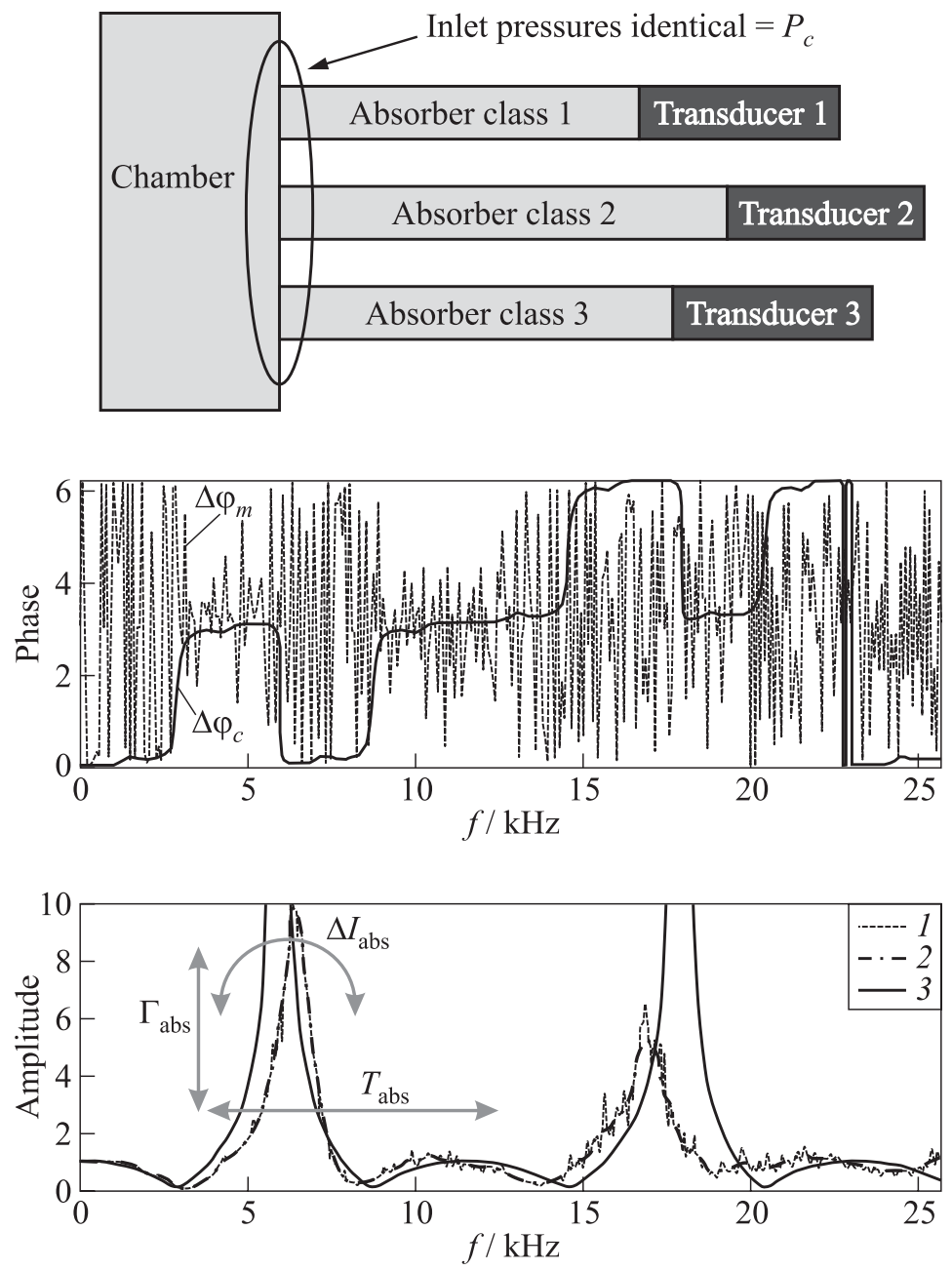

Figure 4 Absorber model validation: $1-\left(A_{0} / A_{i}\right)_{m} ; 2-\left(A_{0} / A_{i}\right)_{m s} ;$ and $3-$ $\left(A_{0} / A_{i}\right)_{c}$

The calculated (solid) curves can be efficiently adjusted to the experimental (dotted) curves for amplitude and phase. The dash-dotted curve represents a filtered version of the dotted amplitude curve. The jumps between zero and $2 \pi$ as well as the oscillatory behavior of the experimental phase curve, deriving from sensor characteristics, are not relevant for the model adaptation. It is noted that a good agreement between STABAN results and test is obviously reached for frequencies below $10 \mathrm{kHz}$. This is the interesting frequency bandwidth for the thrust chamber considered. Beyond this limit, internal absorber effects from 
linear viscous and thermal wall resistances [4] increasingly dominate, since they are proportional to the square root of frequency, making deviations between model and experiment greater:

$$
R_{\mathrm{abs}}=\frac{\Gamma_{\mathrm{abs}}\left|\dot{m}^{\prime}\right|}{\rho_{\mathrm{abs}} A^{2}} .
$$

In STABAN, Eq. (8), which describes the nonlinear resistance from flow losses around the absorber inlet edges, is utilized for model validation in which $\Gamma_{\text {abs }}$ is the resistance factor for flow losses around the absorber inlet. With this value, the solid amplitude curve can be vertically shifted. The effective fluid temperature $T_{\text {abs }}$ is indirectly considered by the fluid properties (like in Eq. (4) for the feed lines) and shifts the solid amplitude curve horizontally. Normally, these parameters are sufficient for model vali-

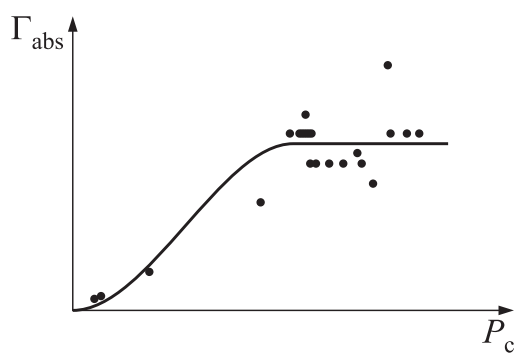

Figure 5 Typical physical law for the absorber resistance factor $\Gamma_{\text {abs }}$ dation purposes. In parallel, also the impact on the thin solid phase curve must be monitored to get the best fit. Figure 5 refers to a thrust chamber with liquid propellants. A physical law could be found for $\Gamma_{\text {abs }}$ for a specific absorber class in the absorber ring.

\section{STABILITY CALCULATIONS}

The applicability range of STABAN is limited to the hardware it is adjusted to. With less accuracy but still valid, it can also be applied inside a family of thrust chambers. Those families are characterized by identical propellant pairs and comparable designs of essential hardware parts. The following main engineering activities are typical for the layout of rocket propulsion systems:

- adjustment of the feed lines to operational requirements and geometrical stage constrains;

- choice of the inner contour of the combustion chamber and the exhaust nozzle;

- decision for the kind of propellants;

- design of the injector, particularly of the injection elements; and

- definition of the operation control from valve opening until main operation stage. 
All of them determine the dynamic behavior of the system in a coupled manner. Still, the most important criteria for the classification into families are the propellants and the design of the injection elements. They must be chosen already at an early development phase. Any modification of propellants or injection elements moves the thrust chamber to another family necessitating a new process of model validation. At late development phases, a severe risk then arises with respect to time schedule and costs.

The other engineering activities named above can be modified without a loss of simulation reliability and without risking family membership, as long as the modifications do not exceed the given validity ranges of the fluid property tables and the physical correlations applied. By changing the feed lines, the inner combustion chamber contour, and the operation control, an optimum solution can be found for a given thrust chamber family for instability risk prevention. Sensitivity studies may accompany to get an imagination how susceptible is the thrust chamber to potential design changes. The dynamic good nature, found out by sensitivity studies, should be a criterion for the choice of future hardware concepts to avoid problems in case of later redesigns.

\subsection{Chugging Analyses}

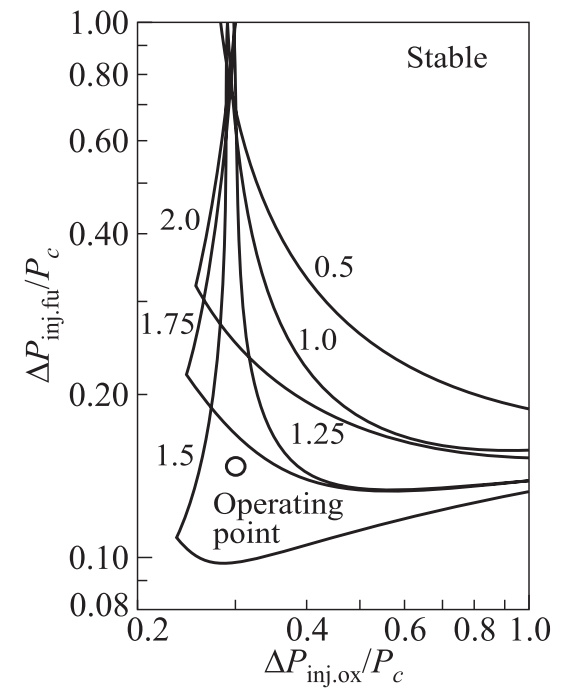

Figure 6 Stability limits for different total combustion time lags
For LF analyses, the feed lines gain more importance at the expense of the combustion chamber which then virtually serves just as a dynamic boundary condition (black box). The increased importance of the feed lines is reflected by a finer distribution of model tubes to consider local effects (see Eq. (4)).

With the terms given in Table 1 for the LF case, Eq. (1) takes the form of the equation presented in [5] for chugging analyses. With this simplified version, stability limit analyses can be performed for various total combustion time lags [5] (Fig. 6). The relative injection pressure drops are taken as the axis values (see also Fig. 9a).

The lines of constant time lag in Fig. 6 are the border lines between stability and instability. When walking clockwise along the lines, the area on the right-hand side is the stable region. On the left side, instability arises. From the calculation results, the interesting fact is that, when increasing the time lag, the stable region extends also to very 
small relative injection pressure drops that is opposite to the common opinion that instability risk rises with increasing time lag. Still, at a certain limit time lag (here: $1.5 \mathrm{~ms}$ ), the situation changes to the opposite and the unstable region again rises. Such limit time lags were first seen in experiments [5] and could not be explained. The theory behind Eq. (1) confirms these experimental findings and, therefore, constitutes a practical plausibility check for STABAN for LF cases.

\subsection{Stability Curves}

Stability curves are the time plots of the system eigenvalue for the HF case. The eigenvalue uniquely expresses whether a system is unstable (positive) or stable (negative).

As an example, Fig. 7 shows plots of the real part of the transfer function. Here, the region above 1 corresponds to instability and the region below 1 to stability. Two tests with a cryogenic thrust chamber, supposed to be identical, are presented. The pressure sensor data of the combustion chamber pressure (static and dynamic sensors) show a short instability in the test of Fig. $7 a$ but
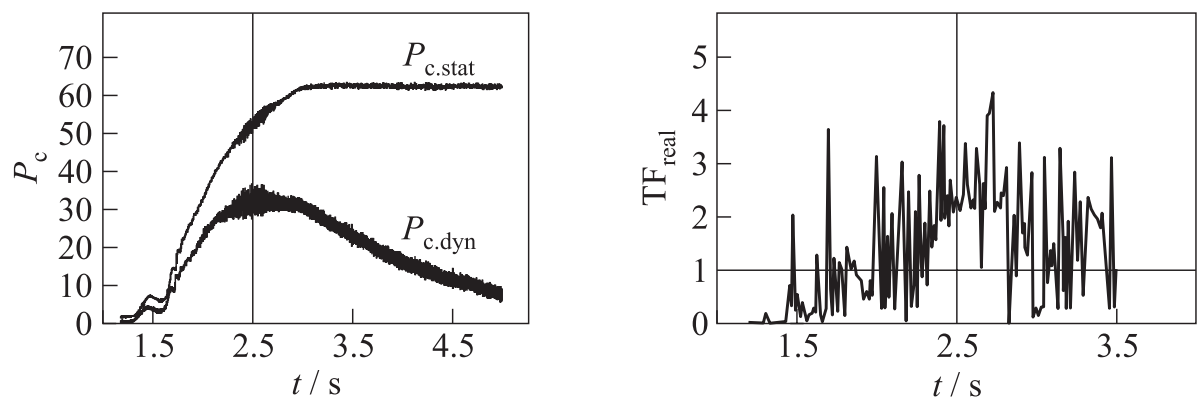

(a)
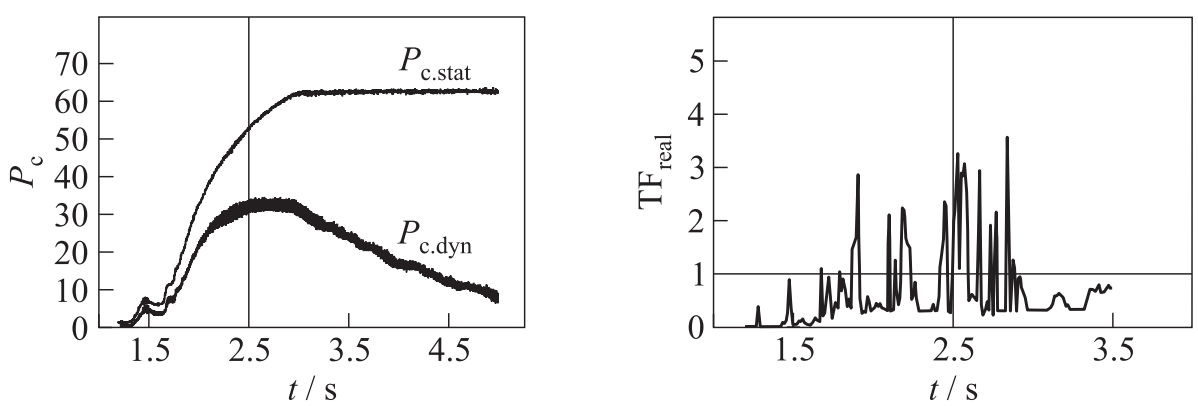

(b)

Figure 7 Stability curves for different dynamic cases (real part of transfer function) 


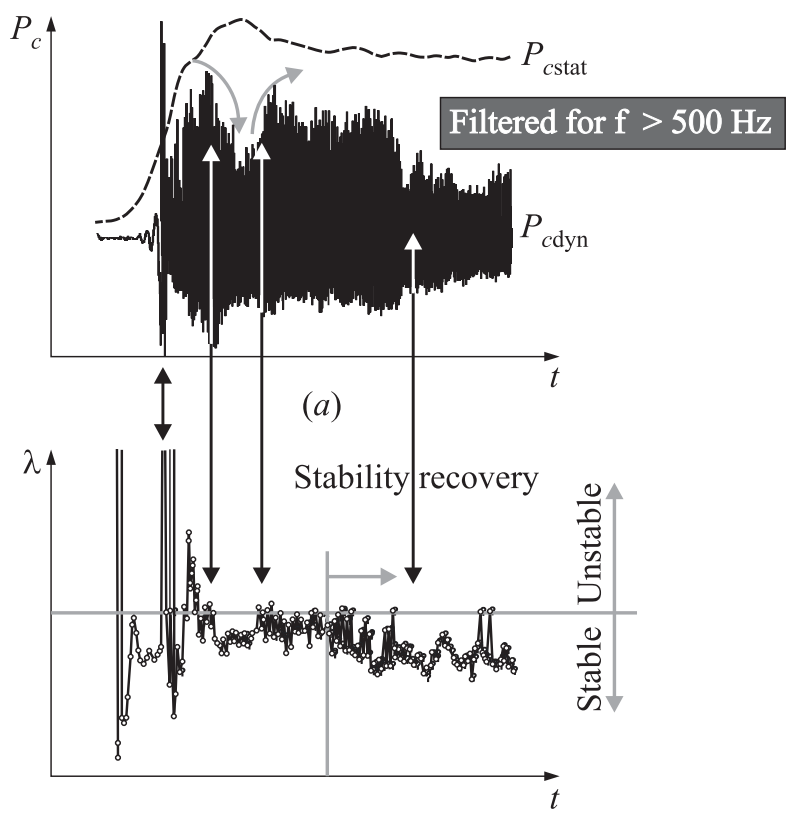

(b)

Figure 8 Stability curve (system eigenvalue)

none in the test of Fig. $7 b$. STABAN was applied to detect hidden reasons for this difference. The calculation results on the right side show that STABAN detects similar situations. For the case of Fig. $7 a$, the unstable range is confirmed (around $t=2.5 \mathrm{~s}$ ). The stable case is also confirmed, since the real part remains below 1.

The steep peaks need not be particularly considered, because they originate from local iteration problems or experimental data inaccuracies (see also Fig. 4).

Another example is given in Fig. 8. Shown in Fig. 8 is the behavior of a thrust chamber with liquid propellants. The stability curve (Fig. $8 b$ ) now shows the system eigenvalue indicating stability if negative or instability if positive. The experimental diagram (Fig. 8a) and the calculation result (see Fig. 8b) are time-correlated to facilitate comparisons. The general statement on STABAN's plausibility from Fig. 7 can be detailed in Fig. 8. After a short peak, the measured dynamic pressure amplitude steadily increases. The starting instability is confirmed by the increase of the eigenvalue to positive values. After having reached the maximum value, the pressure amplitude again reduces to a minimum and finally reaches a limit cycle. In the eigenvalue plot, the situation is fully similar: the eigenvalue falls down to negative (stabilizing) and then reaches limit stability around zero. After the limit cycle, the eigenvalue starts falling down. 
This also makes pressure amplitude decreasing, and the situation becomes stable again.

\subsection{Stability Mappings}

The stability curves are particularly interesting for the detection and explanation of unexpected instability risks in acceptance or qualification tests to define quick countermeasures, be they hardware redesigns or operation control modifications from test to test. Since these calculations require test data like pressures and temperatures, they are not applicable at early development stages, except extrapolations from former development programs are feasible and fulfill the family requirements.

For parameter and sensitivity studies at early stages of development programs, the stability mappings are more suitable [6]. Two parameters are chosen which are considered essential for the elaboration of different design options and scattered around the expected main operation point or the probable startup range. STABAN calculates the stability risk in this field and finally gives a $3 \mathrm{D}$ surface plot. Figure 9 shows an example for a small-scale thruster (Fig. $9 a$ ) with hypergolic propellants and a large-scale thrust chamber (Fig. 9b) with green propellants. Both sides of the diagram refer to the T1 mode.

Figure $9 a$ shows a safe startup (solid curve) up to main stage (triangle). The region critical to instability (dots) is not touched or crossed. The safety margin can be directly determined as the numerical values of the relative injection pressure drops. They are major design criteria for injector layout. The oxidizer and fuel are equally uncritical. The given plot is identical to Fig. 6 with respect to the general shape of the stability limit line.

In Fig. 9b, the oxidizer mass flow (to the right) and the fuel mass flow (to the left) are chosen as the stability parameters. The ordinate gives the system

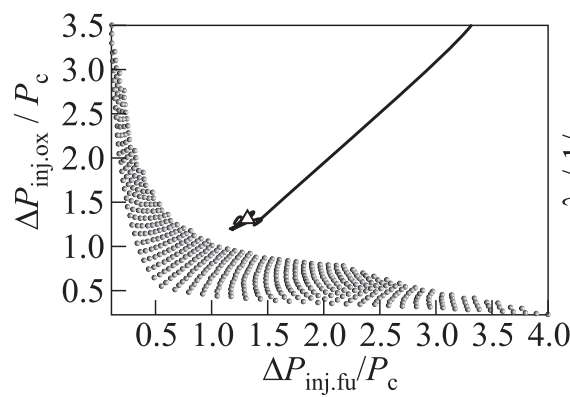

(a)

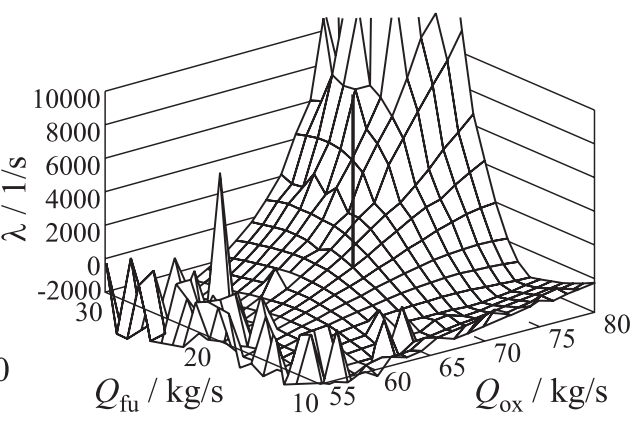

(b)

Figure 9 Stability mapping: (a) relative injection pressure drops; and $(b)$ propellant flow rates 
eigenvalue. Isolated peaks originate from numerical iteration problems and can be neglected. The nominal main operation stage is indicated by the thick black line.

The figure shows the sensitivity of the thrust chamber around this nominal condition. The flatter the area, the less nervous the thrust chamber reacts to operation variations due to physical dispersions or sudden and unexpected events from outside. This means that a prime development goal with respect to instability prevention should be to realize a flat eigenvalue surface below zero around the nominal operation point.

Furthermore, it can be seen that, on the oxidizer side, an increase of mass flow rate continuously deteriorates the safety margin and increases the instability risk. On the fuel side, the situation is different. Going to higher mass flow rates at constant oxidizer flow rate also increases instability risk, but less heavily, and it even seems that the maximum is reached. If the fuel mass flow rate is reduced from nominal, a minimum of the eigenvalue is reached indicating safe operation, but at further reduction, the instability risk increases again. This means that, on fuel side, a clear optimum is given which can potentially be explained by the counteracting physical effects of feed line coupling, depending on injection pressure loss, and propellant jet preparation (total combustion time lag and droplet drag), depending on the injection velocity ratio. If the thrust chamber would be operated at this fuel flow optimum, nearly no dependency on oxidizer side would be given in a wide range of mass flow rate, i. e., the stability risk would be independent from the oxidizer side.

\subsection{Safety Corridors}

Another way of STABAN application is to determine safety corridors. They try to help defining proper startup corridors for the thrust chamber to minimize the risk for crossing critical regions or to optimize the hardware in these corridors that no instability problems can arise.

The safety corridors given in Fig. 10 refer to early investigations for a cryogenic thrust chamber and were applied to the combustion chamber pressure and the propellant mass flow rates as the main operation variables. The instability risk is calculated along these lines and in between for each variable and indicates potential instability risks by black dots along the lines. Thereby, the size of the black dots indicates how far the eigenvalue is above zero (see also Fig. 9b), i. e., how significant the risk for instability is.

From a quick inspection of Fig. 10, it can be derived that chamber pressure is quite uncritical in the chosen corridor in this specific case, whereas the oxidizer and fuel mass flow rates are still critical at the low corridor boundaries and that for oxidizer, more at late startup and for fuel, more at early startup. The mass flow corridors may be newly defined or the hardware partly redesigned to improve the situation. 


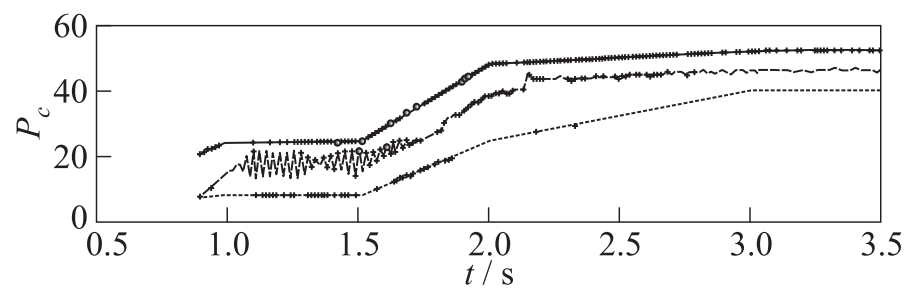

(a)

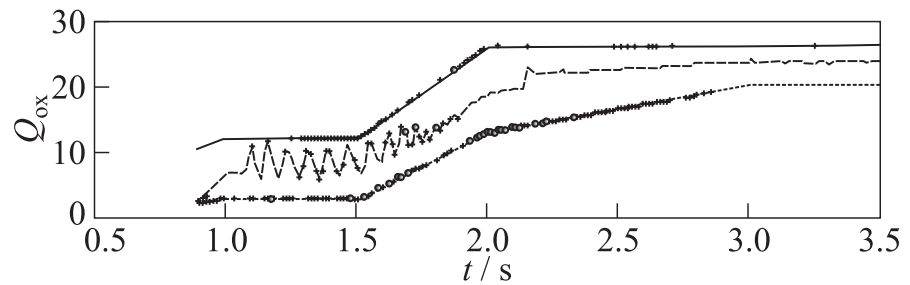

(b)

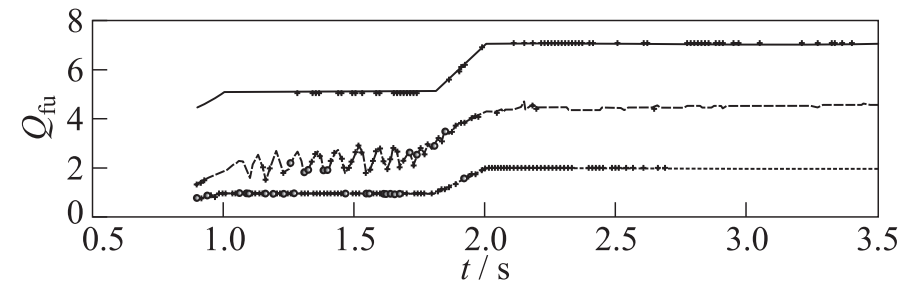

(c)

Figure 10 Safety corridors around thrust chamber startup lines

\section{SUMMARY AND CONCLUDING REMARKS}

STABAN has acquired a solid state of modeling but requires further effort to consolidate and extend the approaches. By a systematic application of STABAN, a new industrial standard can be established for future development programs in the field of instability prevention benefiting from the continuously increasing knowledge in the French-German REST group and the permanently improved model validation level. STABAN can be utilized to define thrust chamber test campaigns, to qualify the hardware against stability specifications, and to provide a stability data base from systematic thrust chamber test analyses. Some application examples show promising perspectives for a general use of STABAN in future thrust chamber development programs in order to reduce the development risk with respect to instability.

The semiempirical kind of STABAN limits its application to certain boundary conditions. Predictions are most reliable for thrust chambers of the same 
family. Families are classified by two prime engineering decisions: the choice of propellants (fluid properties) and the injection element design (dynamic feed line coupling and jet preparation). The propellants and the injection system should be solidly defined already at an early development phase and then kept unchanged not to make later predictions or extrapolations invalid for instability risk estimation or to necessitate additional model validation efforts.

The present paper just introduces the main models of STABAN to keep the volume at reasonable limits. There is a considerable number of accompanying models which are not mentioned or described. Beside others, they account for secondary effects, distributed quantities in the combustion chamber, linear and nonlinear resistance modeling, effective sound velocity treatment and the consideration of measurement adapters.

\section{ACKNOWLEDGMENTS}

The activities for the STABAN development and also Astrium's participation in the French-German REST group are parts of the German TEKAN project which is substantially co-funded by the DLR.

The REST group has essentially contributed to STABAN by the frank and valuable scientific and technical discussions in the past decade and the research results helped to complete it for industrial use. In the REST group, the cooperation with the DLR, Lampoldshausen, is particularly highlighted for their readiness for test data exchange and interest in the modeling progress. Geoff Searby from the IRPHE, Marseilles, has also contributed to the overall understanding and supported the engineering viewpoint of the problem. Particularly, I thank Paul Spagna for his patient explanations and the practical case studies from decades of experience.

\section{REFERENCES}

1. Harrje, D. T., and F.H. Reardon. 1972. Liquid propellant rocket combustion instability. NASA SP-194.

2. Barrère M. 1961. Raketenantriebe: Study book. Elsevier Publ. Co. 450 p.

3. Bell, W. A., and B. T. Zinn. 1973. Computer program to determine the irrotational nozzle admittance. LEW-12019.

4. Searby, G., A. Nicole, M. Habiballah, and E. Laroche. 2008. Prediction of the efficiency of acoustic damping cavities. J. Propul. Power 24(3).

5. Wenzel, L. M., and J.R. Szuch. 1965. Analysis of chugging in liquid-bipropellant rocket engines using propellants with different vaporization rates. NASA TN D3080 .

6. Cavitt, R. C., and R. A. Frederick. 2008. Laboratory scale survey of pentad injector stability characteristics. J. Propul. Power 24(3). 